\title{
BMJ Open Ulcerative colitis outcomes research in Japan: protocol for an observational prospective cohort study of YOURS (YOu and Ulcerative colitis: Registry and Social network)
}

Hajime Yamazaki, ${ }^{1}$ Katsuyoshi Matsuoka, ${ }^{2}$ Jovelle Fernandez, ${ }^{3}$ Toshifumi Hibi, ${ }^{4}$ Mamoru Watanabe, ${ }^{5,6}$ Tadakazu Hisamatsu, $^{7}$ Shunichi Fukuhara ${ }^{1}$

To cite: Yamazaki H,

Matsuoka K, Fernandez J, et al. Ulcerative colitis outcomes research in Japan: protocol for an observational prospective cohort study of YOURS (YOu and Ulcerative colitis: Registry and Social network). BMJ Open 2019;9:e030134. doi:10.1136/ bmjopen-2019-030134

\section{- Prepublication history for} this paper is available online. To view these files, please visit the journal online (http://dx.doi. org/10.1136/bmjopen-2019030134).

Received 28 February 2019

Revised 22 July 2019

Accepted 01 August 2019
Check for updates

(C) Author(s) (or their employer(s)) 2019. Re-use permitted under CC BY-NC. No commercial re-use. See rights and permissions. Published by BMJ.

For numbered affiliations see end of article.

Correspondence to

Hajime Yamazaki;

yamazaki-myz@umin.ac.jp

\section{ABSTRACT}

Introduction Ulcerative colitis (UC) is a chronic inflammatory disease that mainly affects the colon in young patients. Typical symptoms of UC are bloody diarrhoea and faecal urgency, which disturb the quality of life (QOL) of patients, and intractable UC leads to hospitalisation and colectomy. To improve relevant outcomes such as symptoms, QOL and colectomy, many clinical questions need to be resolved regarding what the ideal lifestyle, psychosocial burden and optimal practice patterns are. In this $\mathrm{YOu}$ and UIcerative colitis: Registry and Social network (YOURS) study, we will investigate the effect of lifestyle, psychosocial factors and practice patterns on patient-reported outcomes (PRO), hospitalisation rate and colectomy rate in Japanese patients with UC.

Methods and analysis For this prospective cohort study, we recruited 2006 patients from five hospitals (Tokyo and Chiba; May 2018-January 2019). Patients will be able to access their own data and compare them with summarised data from all patients on the website beyond the YOURS study. At baseline, patients will answer a questionnaire regarding lifestyle (diet, exercise, sleep and work), psychosocial factors (stress, depression and social support) and PRO (symptoms and QOL). Information on practice patterns (eg, medications, endoscopy frequency) will be collected from electronic medical records. Gaps between patients' needs and healthcare professionals' practice will be identified. Follow-up surveys will be conducted periodically for approximately 3 years. Research questions suggested by patients and healthcare professionals may be used in subsequent surveys. Results from the YOURS study will demonstrate optimal UC management strategies to improve relevant outcomes.

\section{ETHICS AND DISSEMINATION}

The study was approved by the ethics committees of five investigational sites before starting the study. The results will be submitted to journals.

\section{TRIAL REGISTRATION NUMBER UMIN000031995.}

\section{Strengths and limitations of this study}

- The YOu and Ulcerative colitis: Registry and Social network (YOURS) study is a large-scale, long-term, prospective observational study to explore optimal ulcerative colitis management for improving relevant outcomes.

- The YOURS study reflects the real clinical setting because any concomitant drugs and therapies are allowed.

- The YOURS study can be expanded for subsequent surveys because patients and healthcare professionals can ask research questions not otherwise included in the survey.

- Patients will be able to access their own data and compare them with summarised data from all patients on the website beyond the YOURS study.

- All investigational sites are located within one region of Japan, which may affect the study results, especially because of lifestyle and psychosocial factors.

\section{INTRODUCTION}

Ulcerative colitis (UC) is a chronic inflammatory disease with no curative treatment. It mainly affects the colon and is associated with bloody diarrhoea and faecal urgency as typical symptoms. ${ }^{1}$ Aberrant immunity in the gut is considered to be involved in the pathogenesis of $\mathrm{UC}^{2}{ }^{2}$ however, the aetiology of UC is not fully understood. UC is increasing around the world, especially in Asia, including Japan. ${ }^{3-5}$ The prevalence of $\mathrm{UC}$ has been reported to range from 5.3 to 63.6 per 100000 people in Asia and from 37.5 to 238 per 100000 people in North America, ${ }^{1}$ and differs between regions, with the prevalence in Japan reported as approximately 100 per 100000 people in $2013 .^{5}$

The onset of UC often has a major influence on patients' quality of life (QOL), and 


\section{Factors}

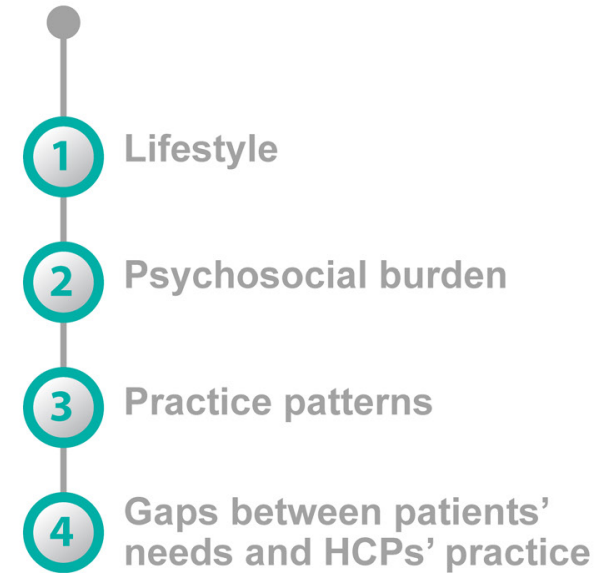

\section{Outcome}

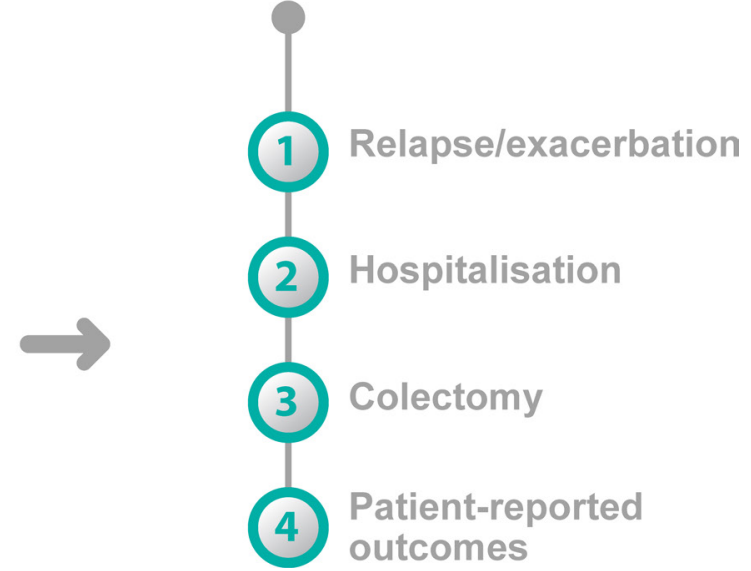

Figure 1 Factors and outcomes assessed in the YOURS study. All four factors can contribute to all four outcomes. HCP, healthcare professional; YOURS, YOu and Ulcerative colitis: Registry and Social network.

intractable UC can lead to hospitalisation and colectomy. Patients need to manage UC throughout their lives with both self-management and support from healthcare professionals (HCPs) to avoid unwanted clinical outcomes, including relapse/exacerbation, hospitalisation, and colectomy, as well as to improve patient-reported outcomes such as QOL.

In this YOURS (YOu and Ulcerative colitis: Registry and Social network) study, we identified four main factors/ challenges to the improvement of patient outcomes: (1) lifestyle, (2) psychosocial factors, (3) practice patterns and (4) gaps between patients' needs and HCPs' practice (figure 1).

First, there has been much discussion and debate about the optimal lifestyle (diet, exercise, sleep and work) for UC management. In particular, diet is a critical issue for both patients and their HCPs. Although a large-scale study has reported that the relationship between diet and relapse is of major interest to patients with inflammatory bowel disease (IBD) and their HCPs ${ }^{6}$ only two exploratory studies have examined the relationship. ${ }^{78}$ However, the results from these studies were not consistent.

Second, psychosocial factors including stress, depression and social support may affect clinical outcomes in UC. Approximately half of patients with IBD have low QOL and depression, ${ }^{9}$ and one-third of patients are reported to feel stigmatised. ${ }^{10}$ It remains unknown how these psychosocial factors affect disease course.

The third challenge is practice patterns. The Selecting Therapeutic Targets in Inflammatory Bowel Disease programme, published in 2015 by IBD specialists, recommends setting concrete treatment goals as part of a 'Treat to Target' strategy. ${ }^{11}$ The agreed treatment goals for UC were clinical remission (defined as resolution of rectal bleeding and diarrhoea/altered bowel habit) and endoscopic remission (based on endoscopic findings). However, achievement of these treatment goals may be accompanied by decreased QOL due to frequent colonoscopies or side effects caused by immune suppression.
Optimal disease monitoring and treatment need to be clarified.

The last challenge is gaps between patients' needs and HCPs' practice. A previous survey has identified important differences between patients and HCPs in their perception of the impact of UC symptoms on patients' lives, suggesting that HCPs may underestimate the effect of UC symptoms on patients. ${ }^{12}$ However, the effect of perception gaps on patient outcomes is unknown.

The YOURS study is an observational prospective cohort study in patients with UC in Japan that aims to clarify how these four challenges affect relapse/exacerbation, hospitalisation, colectomy and patient-reported outcomes such as QOL.

\section{METHODS AND ANALYSIS \\ Study design}

The YOURS study is an observational prospective cohort study with a 3-year follow-up being conducted at five investigational sites in Japan. Patient registration started in May 2018 and ended in January 2019. As of 11 January 2019, 2006 patients have been enrolled.

The study is being conducted in compliance with the Declaration of Helsinki, Ethical Guidelines for Medical Research on Humans, Ministry of Education, Culture, Sports, Science and Technology of Japan and Ministry of Health, Labour and Welfare of Japan and all applicable laws and guidelines. Written informed consent will be obtained from all patients. The study is registered at the University hospital Medical Information Network (UMIN) Center.

\section{Study population and sample size}

Patients diagnosed with UC, as defined by the 'Evidencebased Clinical Practice Guidelines for Inflammatory Bowel Disease', ${ }^{5}$ who are $\geq 16$ years of age at informed consent and are attending the investigational sites are eligible for enrolment. 
The sample size was determined by considering the feasibility of enrolling patients.

\section{Outcome measures and survey items}

Relapse/exacerbation, hospitalisation, colectomy and patient-reported outcomes such as QOL will be assessed as main outcomes. Lifestyle, psychosocial factors, practice patterns and gaps between patients' needs and HCPs' practice will be evaluated as exposures. All surveys will be conducted in Japanese and will include standard, validated questionnaires for most measures of lifestyle, psychosocial factors and symptoms.

At the initial survey, patients will complete written questionnaires with demographic, lifestyle, psychosocial and symptom questions (table 1, figure 2). Following the initial survey, patients will complete a three-item brief symptom survey including psychosocial and symptom questions by smartphone application, email, phone or written questionnaire every 3 months, and a follow-up

Table 1 Survey items/questionnaires

Category Item (questionnaire)

By patients

Initial survey
Patient information Body height, weight, UC history, appendectomy, medical history including malignant tumour, anal fistula and perianal abscess, family history of inflammatory bowel disease, vaccination history, smoking history, education level, economic status and pregnancy history

Lifestyle $\quad$ Exercise (IPAQ), sleep (PSQI) and labour productivity (WPAI)

Psychosocial

factors

Social support (mMOS-SS), medication adherence (ASK-12), comprehensive QOL (QGEN10 survey), disease-specific QOL (SIBDQ), depression/anxiety (HADS) and stress (JPSS)

Symptoms

Stool frequency and rectal bleeding (PRO-2), pain (NRS) and fatigue (FACIT-F)

Others

Research questions not included in this survey

Special survey (in patients in
remission for at least 90 days, at
3 months from the time of initial
survey)

\begin{tabular}{|c|c|c|}
\hline \multirow[t]{2}{*}{$\begin{array}{l}\text { Symptom survey (every } 3 \\
\text { months) }\end{array}$} & $\begin{array}{l}\text { Psychosocial } \\
\text { factors }\end{array}$ & Disease-specific QOL (QDIS-1) \\
\hline & Symptoms & Stool frequency and rectal bleeding (PRO-2) \\
\hline \multirow[t]{4}{*}{ Follow-up survey (every year) } & Lifestyle & Sleep (PSQI) and labour productivity (WPAI) \\
\hline & $\begin{array}{l}\text { Psychosocial } \\
\text { factors }\end{array}$ & $\begin{array}{l}\text { Medication adherence (ASK-12), comprehensive QOL (QGEN10 } \\
\text { survey), disease-specific QOL (SIBDQ), depression/anxiety (HADS) } \\
\text { and stress (JPSS) }\end{array}$ \\
\hline & Symptoms & $\begin{array}{l}\text { Stool frequency and rectal bleeding (PRO-2), pain (NRS) and fatigue } \\
\text { (FACIT-F) }\end{array}$ \\
\hline & Others & Research questions not included in this survey \\
\hline
\end{tabular}

By $H C P$

HCP survey (initial)

Lifestyle Diet (BDHQ)

Others Diet, exercise and stress management strategies to prevent relapse

factors

Psychosocial Medication adherence (ASK-12), comprehensive QOL (QGEN10 survey), disease-specific QOL (SIBDQ), depression/anxiety (HADS) Stool frequency and rectal bleeding (PRO-2), pain (NRS) and fatigue Research questions not included in this survey

\begin{tabular}{lll} 
HCP survey (initial) & - & $\begin{array}{l}\text { Observations related to lifestyle guidance for diet, exercise and } \\
\text { strategies to avoid stress; research questions not included in this } \\
\text { survey }\end{array}$ \\
$\begin{array}{l}\text { Medical record survey (initial } \\
\text { only) }\end{array}$ & $\begin{array}{l}\text { Patient information } \\
\text { Medical record survey (initial, } 3\end{array}$ & $\begin{array}{l}\text { Age, sex, worst disease extent (eg, pancolitis, left-sided colitis, } \\
\text { extensive colitis), extraintestinal complications and treatment history }\end{array}$ \\
$\begin{array}{l}\text { months from the initial survey, } \\
\text { every year) }\end{array}$ & $\begin{array}{l}\text { Partial Mayo score, medication, observation of endoscope and } \\
\text { pathologic observation, Clostridium difficile infection, blood } \\
\text { examination, calprotectin in stool, hospitalisation, colectomy }\end{array}$ \\
\hline
\end{tabular}

ASK-12, ${ }^{15-18}$ Adherence Starts with Knowledge-12; BDHQ, ${ }^{19}{ }^{20}$ Brief-type self-administered Diet History Questionnaire; FACIT-F, ${ }^{21-23}$ Functional Assessment of Chronic Illness Therapy-Fatigue; HADS, ${ }^{24-27}$ Hospital Anxiety and Depression Scale; HCP, healthcare professional; IPAQ, ${ }^{28} 29$ International Physical Activities Questionnaire; JPSS, ${ }^{30} 31$ Japanese version of the Perceived Stress Scale; mMOS-SS, ${ }^{32-34}$ modified Medical Outcomes Study Social Support Survey; NRS, ${ }^{35}$ Numerical Rating Scale; PRO-2, ${ }^{13}$ two-item patient-reported outcomes; PSQI, ${ }^{36}{ }^{37}$ Pittsburgh Sleep Quality Index; QDIS, ${ }^{38}$ Quality of life Disease-specific Impact Scale; QGEN, ${ }^{39}$ Quality of life GENeral; QOL, quality of life; SIBDQ, ${ }^{40-42}$ Short version of Inflammatory Bowel Disease Questionnaire; UC, ulcerative colitis; WPAI, ${ }^{44}$ Work Productivity and Activity Impairment questionnaire. 


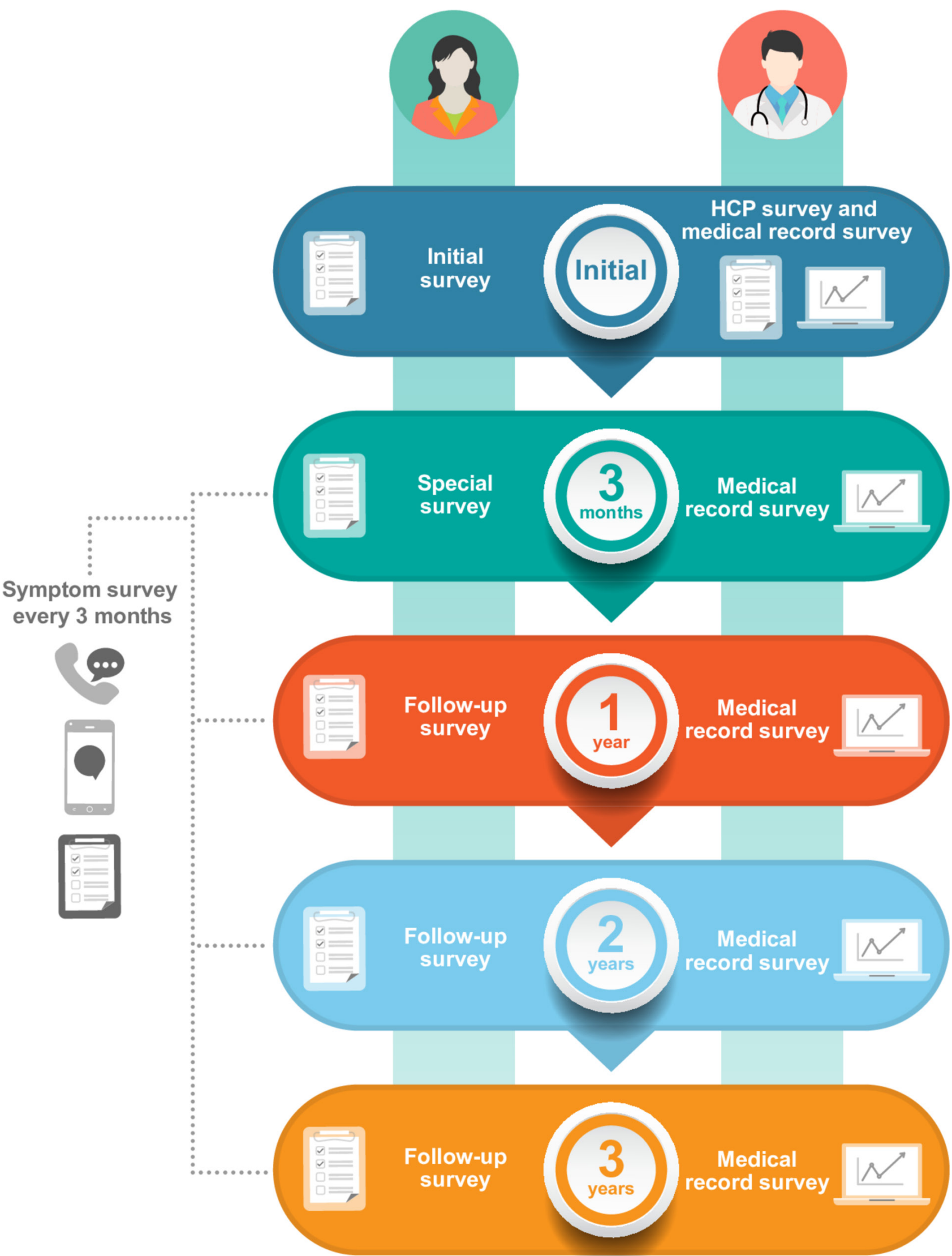

Figure 2 Study design. Surveys on the left side are for patients, and surveys on the right side are for HCPs. All surveys were completed by written questionnaires, except for the symptom survey, which could be completed by smartphone application, email, phone or written questionnaire. The special survey at 3 months from the time of initial survey was only completed by patients who maintained remission for at least 90 days. HCP, healthcare professional. 


\begin{tabular}{ll}
\hline $\begin{array}{l}\text { Table } 2 \\
\text { bleeding }\end{array}$ & Definition of stool frequency score and rectal \\
\hline Score & Definition \\
\hline $\begin{array}{cc}\text { Stool frequency } \\
0\end{array}$ & Normal number of stools \\
1 & $1-2$ stools more than normal \\
2 & $3-4$ stools more than normal \\
3 & 5 or more stools more than normal \\
Rectal bleeding \\
0 & None \\
1 & Streaks of blood with stool less than half the time \\
2 & Obvious blood with stool most of the time \\
3 & Blood alone passed \\
\hline
\end{tabular}

PRO-2, two-item patient-reported outcomes.

survey including lifestyle, psychosocial and symptom questions by written questionnaire every year for 3 years (table 1, figure 2). Patients who maintain remission for at least 90 days will complete a special survey by written questionnaire at 3 months from the time of the initial survey, which includes lifestyle questions focusing on diet, exercise and stress management as strategies to prevent relapse (table 1, figure 2). Remission is defined as a stool frequency score of 0 or 1 and a rectal bleeding score of 0 (two-item patient-reported outcome; table 2) ${ }^{13}$ Patients will have the opportunity to provide their own research questions at the initial survey and all follow-up surveys. We plan to categorise and create a ranking of the research questions. The members of the steering committee will review the ranking of these research questions. If the research questions are feasible for this study, they will be included in the subsequent surveys within the YOURS study. The three-item brief symptom survey will provide the most important information for this study because the survey evaluates relapse or exacerbation of UC. In cases where patients are reluctant to complete the follow-up survey, for reasons such as changing hospitals, we will offer to continue with only the brief symptom survey every 3 months to reduce the number of dropouts.

In the HCP survey, HCPs will complete written questionnaires about their guidance to patients with remission regarding diet, exercise and stress management in order to prevent relapse. Answers from patients and HCPs will be compared and any gaps in the perception of the ideal lifestyle to prevent relapses will be identified and explored. In addition, HCPs will collect patients' medical information such as medication use, blood examinations, stool examinations, endoscopic findings, pathologic findings, hospitalisations, colectomy status and adverse events (throughout the study) from electronic medical records at all surveys (table 1 , figure 2 ).

\section{Data management}

The YOURS study uses an electronic data capture system to register patients, collect survey answers and create a database, which can be accessed securely by only those investigators who have received appropriate training. The principal investigators are responsible for ensuring data quality.

\section{Feedback to patients}

Patients will be provided written feedback on their data compared with the other patients participating in the YOURS study. Moreover, patients can use the website for reviewing their data over time, comparing with others and sharing their data with HCPs via the website, provided the patients have requested access and agreed to the transfer of their data from the database to the website with written informed consent (figure 3).

\section{Statistical analysis}

Analysis groups will be determined for each outcome measure and survey item. The baseline data on demographics, lifestyle, psychosocial factors, practice patterns and gaps between patients' needs and HCPs' practice will be summarised. The following statistical analyses to clarify the association of these baseline data with relapse/exacerbation, hospitalisation, colectomy and patient-reported outcomes such as QOL will be conducted.

Logistic regression analysis or Cox proportional hazard model will be used for analysis of binary variables. Linear regression analysis will be used for analysis of continuous variables. Multilevel analysis may be used for considering differences between investigational sites. Confounding factors will be adjusted appropriately for all analyses.

Interim database locks and interim analyses are planned as follows: initial survey, special survey at 3 months from the time of the initial survey, and follow-up surveys at 2 and 3 years after the initial survey.

\section{Patient and public involvement statement}

We will collect research questions from both patients and HCPs from surveys in the YOURS study. If the research questions are feasible for this study, they will be included in the subsequent surveys within the YOURS study.

\section{ETHICS AND DISSEMINATION}

The YOURS study is sponsored by Takeda Pharmaceutical Company Limited (Tokyo, Japan) and advice on the study plan was obtained from the Japanese Society for Inflammatory Bowel Disease. The study is managed by six joint research organisations, including Takeda and five investigational sites: Tokyo Medical and Dental University, Medical Hospital (Tokyo, Japan); Kitasato University Kitasato Institute Hospital (Tokyo, Japan); Kyorin University Hospital (Tokyo, Japan); Tokyo Women's Medical University Hospital (Tokyo, Japan) and Toho University Sakura Medical Center (Chiba, Japan), and was approved by the ethics committees of five investigational sites before starting the study. The YOURS study has a steering committee consisting of medical experts conducting 


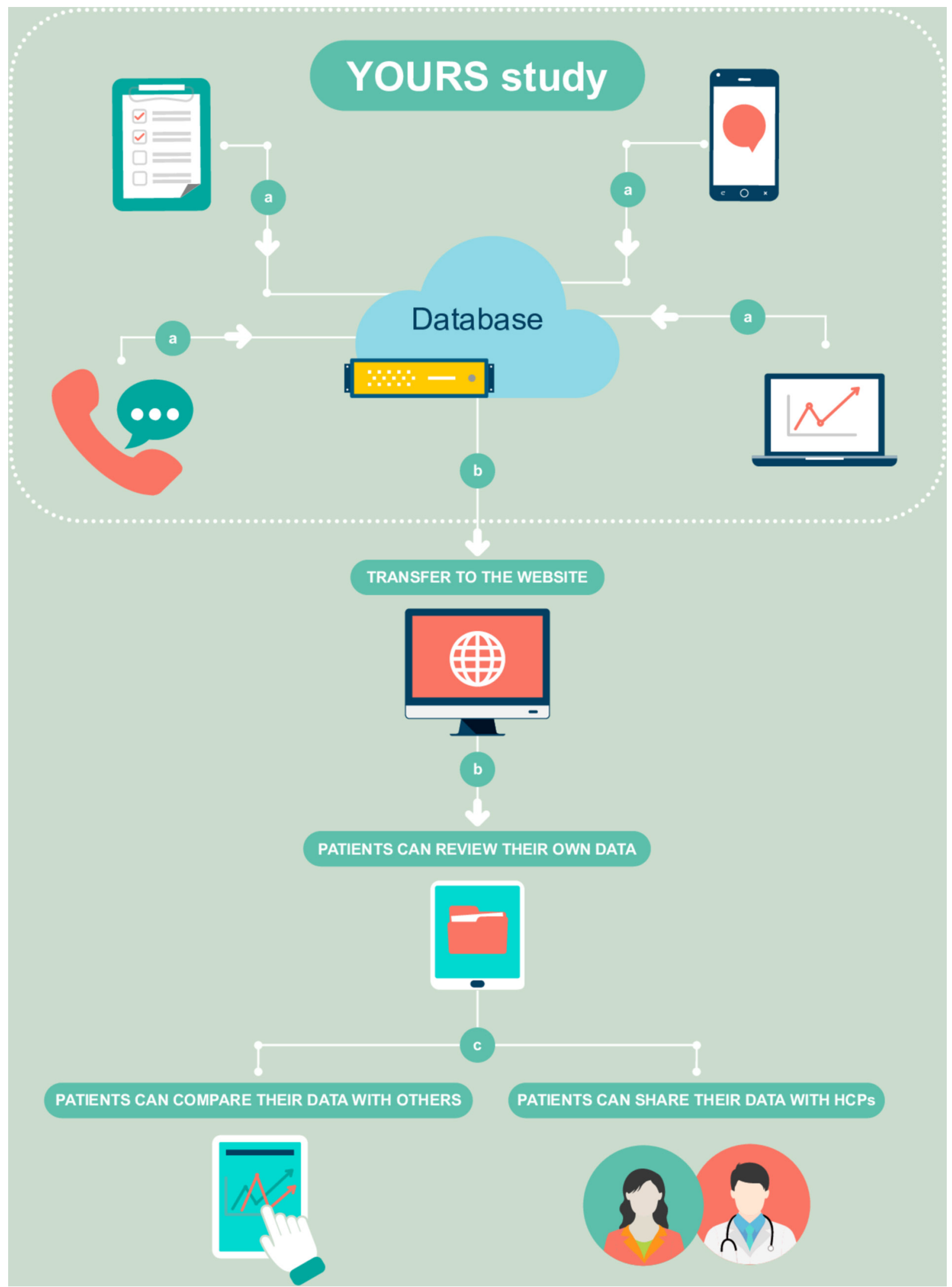

Figure 3 Feedback scheme of the YOURS study. (a) Survey answers and medical records will be collected from patients and HCPs by written questionnaire, smartphone application, email or phone, and stored in the YOURS study database. (b) Patients can use a website for reviewing their own data over time if the patients have requested access and agreed to the transfer of their data to the website. (c) Patients can also use the website for comparing their data with others, and for sharing their data with HCPs via the website. HCP, healthcare professional; YOURS, YOu and Ulcerative colitis: Registry and Social network. 
the study, representatives of the joint research organisations and clinical epidemiology experts. The roles of the steering committee are to supervise the overall study operation, provide medical expertise guidance, ensure the scientific quality of the study is at a high level and update the protocol appropriately when needed. The results will be submitted to journals for publication.

\section{DISCUSSION}

There are four primary goals of the YOURS study: (1) develop a platform to share clinical knowledge and patient experience, (2) define optimal care for UC, (3) fill gaps between patients' needs and HCPs' practice and (4) improve relevant outcomes. To achieve these goals, the YOURS study will investigate how four main challenges (lifestyle, psychosocial factors, practice patterns and gaps between patients' needs and HCPs' practice) affect relapse/exacerbation, hospitalisation, colectomy and patient-reported outcomes such as QOL.

Using the YOURS study results, we plan to create a booklet to clarify how challenges such as lifestyle, psychosocial factors, practice patterns and gaps between patients' needs and HCPs' practice affect clinical outcomes. Although booklets are a preferred adjunct source of information for patients with IBD and it is important to distribute booklets, the same information will also be disseminated in other ways, such as websites, as needed. ${ }^{14}$ The YOURS study booklet will include specific information on diet, exercise, sleep, work, stress, depression and social support. Moreover, if we identify any gaps between patients' needs and HCPs' practice regarding lifestyle guidance, we will include that information in the booklet as background information. We anticipate that the booklet will facilitate the sharing of information between patients and HCPs, thereby helping to bridge gaps between patients' needs and HCPs' practice.

The YOURS study is a large-scale, long-term, prospective observational study without any prohibited concomitant drugs and therapies, thereby reflecting real clinical settings. However, as all investigational sites are located in Tokyo or Chiba (which is near Tokyo) in Japan, the applicability of the study results to other locations may be affected by lifestyle and psychosocial factors influenced by regional, country and/or cultural differences. The other limitation of this study is the possibility that patients will confuse some of the questions, because various kinds of scales are used in each survey to evaluate lifestyle and psychosocial factors. The YOURS study can potentially be expanded in subsequent surveys within the study because patients and HCPs can ask research questions not otherwise included in the survey.

In conclusion, the YOURS study provides an important opportunity to clarify how challenges such as lifestyle, psychosocial factors, practice patterns and gaps between patients' needs and HCPs' practice affect clinical outcomes in patients with UC in Japan. We anticipate that results from the YOURS study will demonstrate optimal UC management strategies to improve relevant outcomes.

\section{Author affiliations}

${ }^{1}$ Department of Healthcare Epidemiology, School of Public Health in the Graduate School of Medicine, Kyoto University, Kyoto, Japan

${ }^{2}$ Division of Gastroenterology and Hepatology, Department of Internal Medicine, Toho University Sakura Medical Center, Chiba, Japan

${ }^{3}$ Japan Medical Office, Takeda Pharmaceutical Company Limited, Tokyo, Japan ${ }^{4}$ Center for Advanced IBD Research and Treatment, Kitasato University Kitasato Institute Hospital, Tokyo, Japan

${ }^{5}$ Department of Gastroenterology and Hepatology, Tokyo Medical and Dental University, Tokyo, Japan

${ }^{6}$ TMDU Advanced Research Institute, Tokyo Medical and Dental University, Tokyo, Japan

${ }^{7}$ Department of Gastroenterology and Hepatology, Kyorin University School of Medicine, Tokyo, Japan

Acknowledgements The authors would like to thank the Japanese Society for Inflammatory Bowel Disease for cooperation to the study. We also thank all patients and healthcare professionals involved in the study.

Contributors All authors participated in the interpretation of study results, and in the drafting, critical revision and approval of the final version of the manuscript. $\mathrm{HY}, \mathrm{KM}, \mathrm{JF}$ and SF were involved in the study design. KM, TH, MW and TH are investigators and have collected the data in the study.

Funding Clinical experts, clinical epidemiologists and Takeda Pharmaceutical Company Limited (Takeda) discussed and developed the protocol, and Takeda contracted out the collection of supporting data by clinicians to site management organisations. Takeda will also contract out data analysis to a third organisation. Regarding manuscript preparation, Jovelle Fernandez, an employee of Takeda Japan Medical Office, is one of the authors of this manuscript. She participated in the study design, interpretation of study results, drafting, critical revision and approval of the final version of the manuscript, as stated in the authors' contributions section. Takeda contracted out medical writing to Yukiko Homma, MPharm and Rebecca Lew, PhD, CMPP of ProScribe: Envision Pharma Group. ProScribe's services complied with international guidelines for Good Publication Practice (GPP3).

Disclaimer Takeda Pharmaceutical Company Limited has been or will be involved in the study design, data collection, data analysis and preparation of the manuscript.

Competing interests KM has received grants and personal fees from Sekisui Medical, AbbVie and Thermofisher Scientific, grants from Alfresa Pharma Corporation, personal fees from Mitsubishi-Tanabe Pharma, Eisai, Kyorin Pharmaceutical, Asahi Kasei Medical, Mochida Pharmaceutical, Kissei, Kyowa Hakko Kirin, Takeda Pharmaceutical, Covidien, Janssen Pharmaceutical, Pfizer, EA Pharma, Astellas, Zeria Pharmaceutical, Celltrion and JIMRO. JF is an employee of Takeda Pharmaceutical Company Limited and owns restricted stocks of Takeda Pharmaceutical Company Limited and GlaxoSmithKline. TH has received grants and personal fees from AbbVie GK, JIMRO Co., EA Pharma and Zeria Pharmaceutical, grants from Otsuka Pharmaceutical Co., personal fees from Mitsubishi-Tanabe Pharm, Kyorin Pharmaceutical, Janssen, Mochida Pharmaceutical, Takeda Pharmaceutical, Gilead Sciences, Celltrion, Nippon Kayaku, Kissei Pharmaceutical, Miyarisan Pharmaceutical, Ferring Pharmaceutical, Eli Lilly, Pfizer Japan and Nichi-Iko Pharmaceutical. MW has received grants and personal fees from Ajinomoto Pharma Co., Kyorin Pharmaceutical Co., Mitsubishi Tanabe Pharma Co., Takeda Pharmaceutical Co., Eisai Co., Otsuka Pharma Co., Kyowa Hakko Kirin Co., Zeria Pharmaceutical Co., JIMRO Co., Asahi Kasei Kuraray Medical Co., EA Pharma Co., AbbVie GK, Astellas Pharma, Daiichi Sankyo Co., Chugai Pharmaceutical Co., Gilead Sciences, Nippon Kayaku Co., Mochida Pharmaceutical Co., Janssen Pharmaceutical K.K., Celltrion Healthcare Co., Pfizer Japan and Miyarisan Pharmaceutical Co.; grants from Toray Industries, MSD K.K., Bristol-Myers K.K., Taiho Pharmaceutical Co., Ayumi Pharmaceutical Co. and Shionogi \& Co. and personal fees from Kissei Pharmaceutical Co., Nichi-lko Pharmaceutical Co., Celgene and Novartis. TH has received grants and personal fees from Mitsubishi Tanabe Pharma, EA Pharma, AbbVie GK, JIMRO, Zeria, Kyorin Pharmaceutical, Takeda Pharmaceutical, Pfizer Japan and Mochida Pharmaceutical; grants from Asahi Kasei Kuraray Medical, Daiichi-Sankyo, Nippon Kayaku and Astellas; and personal fees from Celgene, Janssen Pharmaceutical and Nichi-Iko Pharmaceutical. SF has received lecture fees from Takeda Pharmaceutical Co., Chugai Pharmaceutical Co., Kyowa Hakko Kirin Co., Daiichi Sankyo Co., Bayer Yakuhin, Otsuka Pharmaceutical Co., Pfizer Japan and MSD K.K.

Patient consent for publication Not required. 
Ethics approval The study was approved by the ethics committees of five investigational sites.

Provenance and peer review Not commissioned; externally peer reviewed.

Data availability statement There are no data in this work.

Open access This is an open access article distributed in accordance with the Creative Commons Attribution Non Commercial (CC BY-NC 4.0) license, which permits others to distribute, remix, adapt, build upon this work non-commercially, and license their derivative works on different terms, provided the original work is properly cited, appropriate credit is given, any changes made indicated, and the use is non-commercial. See: http://creativecommons.org/licenses/by-nc/4.0/.

\section{REFERENCES}

1. da Silva BC, Lyra AC, Rocha R, et al. Epidemiology, demographic characteristics and prognostic predictors of ulcerative colitis. World $\mathrm{J}$ Gastroenterol 2014;20:9458-67.

2. Lee SH, Kwon JE, Cho M-L. Immunological pathogenesis of inflammatory bowel disease. Intest Res 2018;16:26-42.

3. Molodecky NA, Soon IS, Rabi DM, et al. Increasing incidence and prevalence of the inflammatory bowel diseases with time, based on systematic review. Gastroenterology 2012;142:46-54. e42.

4. Japan Intractable Diseases Information Center. Annual report 20122016 [in Japanese]. Available: http://www.nanbyou.or.jp/entry/1356 [Accessed 28 December 2018].

5. Matsuoka K, Kobayashi T, Ueno F, et al. Evidence-based clinical practice guidelines for inflammatory bowel disease. J Gastroenterol 2018:53:305-53.

6. Hart AL, Lomer M, Verjee A, et al. What are the top 10 research questions in the treatment of inflammatory bowel disease? A priority setting partnership with the James Lind alliance. J Crohns Colitis 2017;11:204-11.

7. Jowett SL, Seal CJ, Pearce MS, et al. Influence of dietary factors on the clinical course of ulcerative colitis: a prospective cohort study. Gut 2004;53:1479-84.

8. Barnes EL, Nestor M, Onyewadume L, et al. High dietary intake of specific fatty acids increases risk of flares in patients with ulcerative colitis in remission during treatment with aminosalicylates. Clin Gastroenterol Hepatol 2017;15:1390-6.

9. Williet N, Sarter H, Gower-Rousseau C, et al. Patient-reported outcomes in a French nationwide survey of inflammatory bowel disease patients. J Crohns Colitis 2017;11:165-74.

10. Taft TH, Ballou S, Keefer L. A preliminary evaluation of internalized stigma and stigma resistance in inflammatory bowel disease. $J$ Health Psychol 2013;18:451-60.

11. Peyrin-Biroulet L, Sandborn W, Sands BE, et al. Selecting therapeutic targets in inflammatory bowel disease (STRIDE): determining therapeutic goals for treat-to-target. Am J Gastroenterol 2015;110:1324-38

12. Schreiber S, Panés J, Louis E, et al. Perception gaps between patients with ulcerative colitis and healthcare professionals: an online survey. BMC Gastroenterol 2012;12:108.

13. Lewis JD, Chuai S, Nessel L, et al. Use of the noninvasive components of the Mayo score to assess clinical response in ulcerative colitis. Inflamm Bowel Dis 2008;14:1660-6.

14. Wong S, Walker JR, Carr R, et al. The information needs and preferences of persons with longstanding inflammatory bowe disease. Can J Gastroenterol 2012;26:525-31.

15. Hahn SR, Park J, Skinner EP, et al. Development of the ASK-20 adherence barrier survey. Curr Med Res Opin 2008;24:2127-38.

16. Matza LS, Park J, Coyne KS, et al. Derivation and validation of the ASK-12 adherence barrier survey. Ann Pharmacother 2009;43:1621-30.

17. Kimura Y, Aoki A, Takeuchi $\mathrm{H}$, et al. Efficacy of the adherence starts with knowledge 12 (ASK-12) questionnaire Japanese version in Japanese actual clinical setting. Am J Respir Crit Care Med 2016;193:A2680.

18. Takemura M, Nishio M, Fukumitsu K, et al. Optimal cut-off value and clinical usefulness of the adherence starts with Knowledge-12 in patients with asthma taking inhaled corticosteroids. J Thorac Dis 2017;9:2350-9.

19. Kobayashi S, Murakami K, Sasaki S, et al. Comparison of relative validity of food group intakes estimated by comprehensive and brief-type self-administered diet history questionnaires against 16 D dietary records in Japanese adults. Public Health Nutr 2011:14:1200-11.

20. Kobayashi S, Honda S, Murakami K, et al. Both comprehensive and brief self-administered diet history questionnaires satisfactorily RANK nutrient intakes in Japanese adults. J Epidemiol 2012;22:151-9.
21. Cella D, Lai J-S, Stone A. Self-Reported fatigue: one dimension or more? lessons from the functional assessment of chronic illness Therapy-Fatigue (FACIT-F) questionnaire. Support Care Cancer 2011;19:1441-50.

22. Tinsley A, Macklin EA, Korzenik JR, et al. Validation of the functional assessment of chronic illness therapy-fatigue (FACIT-F) in patients with inflammatory bowel disease. Aliment Pharmacol Ther 2011;34:1328-36.

23. Yellen SB, Cella DF, Webster K, et al. Measuring fatigue and other anemia-related symptoms with the functional assessment of cancer therapy (fact) measurement system. J Pain Symptom Manage 1997;13:63-74

24. Zigmond AS, Snaith RP. The hospital anxiety and depression scale. Acta Psychiatr Scand 1983;67:361-70.

25. Zigmond AS, Snaith RP. Hospital anxiety and depression scale (HAD scale). Seishinka Shindangaku 1993:371-2.

26. Yamamoto-Furusho JK, Sarmiento-Aguilar A, García-Alanis M, et al. Hospital anxiety and depression scale (HADS): validation in Mexican patients with inflammatory bowel disease. Gastroenterol Hepatol 2018;41:477-82.

27. Matsudaira $\mathrm{T}$, Igarashi $\mathrm{H}$, Kikuchi $\mathrm{H}$, et al. Factor structure of the hospital anxiety and depression scale in Japanese psychiatric outpatient and student populations. Health Qual Life Outcomes 2009;7:42.

28. Craig CL, Marshall AL, Sjöström M, et al. International physical activity questionnaire: 12 -country reliability and validity. Med Sci Sports Exerc 2003;35:1381-95.

29. Murase N, Katsumura T, Ueda C, et al. Validity and reliability of Japanese version of international physical activity questionnaire. Kousei no Shihyo 2002;49:1-9.

30. Cohen S, Kamarck T, Mermelstein R. A global measure of perceived stress. J Health Soc Behav 1983;24:385-96.

31. Mimura C, Griffiths P. A Japanese version of the perceived stress scale: translation and preliminary test. Int J Nurs Stud 2004;41:379-85.

32. Sherbourne CD, Stewart AL. The MOS social support survey. Soc Sci Med 1991;32:705-14.

33. Moser A, Stuck AE, Silliman RA, et al. The eight-item modified medical outcomes study social support survey: psychometric evaluation showed excellent performance. J Clin Epidemiol 2012;65:1107-16.

34. Togari T, Yokoyama Y. Application of the eight-item modified medical outcomes study social support survey in Japan: a national representative cross-sectional study. Qual Life Res 2016;25:1151-8.

35. Williamson A, Hoggart B. Pain: a review of three commonly used pain rating scales. J Clin Nurs 2005;14:798-804.

36. Buysse DJ, Reynolds CF, Monk TH, et al. The Pittsburgh sleep quality index: a new instrument for psychiatric practice and research. Psychiatry Res 1989;28:193-213. 3rd.

37. Doi Y, Minowa M, Uchiyama M, et al. Psychometric assessment of subjective sleep quality using the Japanese version of the Pittsburgh sleep quality index (PSQI-J) in psychiatric disordered and control subjects. Psychiatry Res 2000;97:165-72.

38. Ware JE, Gandek B, Guyer R, et al. Standardizing disease-specific quality of life measures across multiple chronic conditions: development and initial evaluation of the QOL disease impact scale (QDIS $®)$. Health Qual Life Outcomes 2016;14:84.

39. Ware JE, Gandek B. Measuring physical and emotional health with Quality of Life General (QGEN囚) Surveys: a user guide for single-item short forms. 2nd edn. Watertown, MA: John Ware Research Group, 2019.

40. Irvine EJ, Zhou Q, Thompson AK. The short inflammatory bowel disease questionnaire: a quality of life instrument for community physicians managing inflammatory bowel disease. CCRPT Investigators. Canadian Crohn's relapse prevention trial. Am J Gastroenterol 1996;91:1571-8.

41. Casellas F, Alcalá M-J, Prieto L, et al. Assessment of the influence of disease activity on the quality of life of patients with inflammatory bowel disease using a short questionnaire. Am J Gastroenterol 2004;99:457-61.

42. Sakurai T. Quality of life in the patients with inflammatory bowel disease. Nihon Rinsho 2012;70(Suppl 1):561-6.

43. Reilly MC, Zbrozek AS, Dukes EM. The validity and reproducibility of a work productivity and activity impairment instrument. Pharmacoeconomics 1993;4:353-65.

44. Gibson PR, Vaizey C, Black CM, et al. Relationship between disease severity and quality of life and assessment of health care utilization and cost for ulcerative colitis in Australia: a cross-sectional, observational study. J Crohns Colitis 2014;8:598-606. 